\title{
The age-metallicity relation with RAVE and TGAS
}

\author{
Jennifer Wojno ${ }^{1}$, Georges Kordopatis ${ }^{2}$, Matthias Steinmetz ${ }^{1}$, \\ Paul J. McMillan ${ }^{3}$ and the RAVE collaboration \\ ${ }^{1}$ Leibniz Institut für Astrophysik Potsdam, Potsdam, Germany \\ ${ }^{2}$ Laboratoire Lagrange, Université Côte d'Azur, Observatoire de la Côte d'Azur, Nice, France \\ ${ }^{3}$ Lund Observatory, Lund University, Lund, Sweden
}

\begin{abstract}
Using RAVE data release 5 (DR5), we explore the age and chemistry of a sample of $\sim 25,000$ FGK turnoff stars in the extended solar neighbourhood $(7<\mathrm{R}<9 \mathrm{kpc})$, by separating our sample into two chemical disc components, and investigating the nature of the age-metallicity relation for both. Overall, we find a flat trend in $[\mathrm{Fe} / \mathrm{H}]$ as a function of age for our $\alpha$-low disc, and a correlation between age and metallicity for the oldest $\alpha$-high stars, confirming age-metallicity trends found in more local, high-resolution studies now for a larger volume. We also find a positive gradient in $[\mathrm{Mg} / \mathrm{Fe}]$ as a function of age for our oldest stars. These results have implications for models which include dynamical evolutionary processes such as radial migration.
\end{abstract}

Keywords. stars: ages, stars: abundances, Galaxy: formation

\section{Introduction}

Determining the age of field stars has long remained a significant hurdle in relating stellar evolution with internal Galactic evolutionary processes to reconstruct the chemodynamical history of our Galaxy. In particular, understanding the local age-metallicity relation is a crucial ingredient in accurately modeling stellar populations in the solar neighbourhood. With improvements to isochrone fitting techniques, and parallaxes available via the Tycho-Gaia Astrometric Solution (TGAS, Lindegren et al. 2016), ages of field stars can be reasonably estimated for a large sample of solar neighbourhood stars. Ages are determined using an updated Bayesian method described in McMillan et al. (2017, this volume), taking TGAS parallaxes as a prior, together with $T_{\text {eff }}, \log g,[\mathrm{M} / \mathrm{H}]$ from RAVE (Steinmetz et al. 2006), and an underlying Galactic model.

\section{Sample selection and chemical separation of disc components}

For this study, we select a local (distance $<1 \mathrm{kpc}$ ), high-quality $(\mathrm{SNR}>60$ ) sample of turnoff stars from RAVE DR5 (Kunder et al. 2017). Our selection criteria in $\mathrm{T}_{\text {eff }}-\log g$ space is shown in Fig. 1 by the dashed red lines. Our final sample consists of 25,017 stars. To our sample of turn-off stars, we apply the probabilistic chemical separation method described in Wojno et al. (2016). This method uses a model metallicity distribution function and $[\alpha / \mathrm{Fe}]$-distribution function for both the $\alpha$-low (thin disc) and $\alpha$-high (thick disc) to determine membership likelihood for each component.

\section{Age-metallicity and age- $\alpha$ relations}

Age-metallicity relation. We find different age-metallicity relations (AMRs) for our two chemical disc components. Our thin disc is consistent with a flat trend, i.e., no correlation between age and metallicity (Fig. 1). In contrast, we find a correlation between age and 

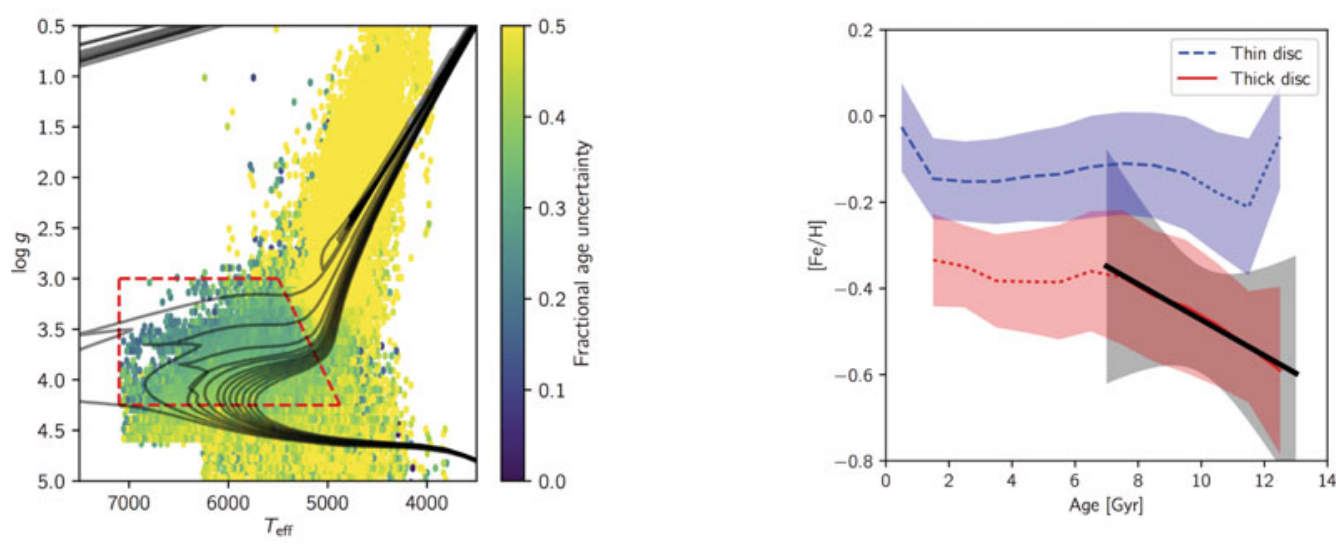

Figure 1: Left: $T_{\text {eff }}-\log g$ diagram showing our parameter space selection (red dashed lines). Bins are colour-coded by their average fractional age uncertainties. Solar metallicity isochrones from 0 to 13 Gyr, in 1 Gyr steps, are plotted in black.

Right: The AMR for our selected thin (dashed blue) and thick (solid red) disc stars. Dotted lines indicate regions were we assume strong contamination between disc components. The black solid line shows the fit used to estimate the AMR for the oldest thick disc stars.

metallicity for our thick disc. When we consider the oldest stars $(\tau>8$ Gyr $)$ in the thick disc, we measure a gradient of $-0.05 \pm 0.08 \mathrm{dex} \mathrm{Gyr}^{-1}$. This falls between trends found by high-resolution studies ( $\sim-0.2$ dex $\mathrm{Gyr}^{-1}$, e.g. Haywood et al. 2013, Bensby et al. 2014), and the recent study by Fuhrmann et al. $2017\left(\sim-0.017\right.$ dex $\left.\mathrm{Gyr}^{-1}\right)$.

Age- $[\alpha / \mathrm{Fe}]$ relation. For the age- $[\alpha / \mathrm{Fe}]$ (here, $[\mathrm{Mg} / \mathrm{Fe}]$ ) relation, we find a flat trend for young stars $(\tau<8 \mathrm{Gyr})$, and a slight positive trend $\left(0.02 \pm 0.04 \mathrm{dex} \mathrm{Gyr}^{-1}\right)$ for the oldest stars. This trend is more shallow than those found by Bensby et al. (2014) and Haywood et al. $(2015)\left(\sim 0.06\right.$ and $\sim 0.05$ dex $\mathrm{Gyr}^{-1}$, respectively), indicating a weaker correlation between age and $[\alpha / \mathrm{Fe}]$ for our chemical thick disc, possibly due to contamination as a result of large age uncertainties. However, we do find a knee at $\sim 8$ Gyr as in the thick disc AMR, which clearly illustrates that the chemical thin and thick discs have experienced distinctly different enrichment histories (e.g. Haywood et al. 2013).

\section{References}

Bensby, T., Feltzing, S., \& Oey, M. S. 2014, A\&A, 562, A71

Binney, J., Burnett, B., Kordopatis, G., et al. 2014, MNRAS, 437, 351

Fuhrmann, K., Chini, R., Kaderhandt, L., \& Chen, Z. 2017, MNRAS, 464, 2610

Haywood, M., Di Matteo, P., Lehnert, M. D., Katz, D., \& Gómez, A. 2013, A\&\&A, 560, A109

Haywood, M., Di Matteo, P., Snaith, O., \& Lehnert, M. D. 2015, A\&A, 579, A5

Kunder, A., Kordopatis, G., Steinmetz, M., et al. 2017, AJ, 153, 75

Lindegren, L., Lammers, U., Bastian, U., et al. 2016, A\& A, 595, A4

Steinmetz, M., Zwitter, T., Siebert, A., et al. 2006, AJ, 132, 1645

Wojno, J., Kordopatis, G., Steinmetz, M., et al. 2016, MNRAS, 461, 4246

This work has made use of data from the European Space Agency (ESA) mission Gaia (https://www. cosmos.esa. int/gaia), processed by the Gaia Data Processing and Analysis Consortium (DPAC, https://www.cosmos.esa.int/ web/gaia/dpac/consortium). Funding for the DPAC has been provided by national institutions, in particular the institutions participating in the Gaia Multilateral Agreement. 